\title{
Unio Internationalis Contra Cancrum U.I.C.C.
}

Listes officiellesdes personnalités élues membresdu comité exécutif, des commissions et comités de I'union

Comite executif j Executive Committee President:

Prof. J. H. Maisin (Belgium). President-elect:

Prof. V. R. Khanolkar (India).

Vice-Presidents:

Asia: Dr. T. Yoshida (Japan).

British Commonwealth:

Dr. 0. H. Warwick (Canada). Europe: Dr. Leiv Kreyberg (Norway). Latin America: Prof. A.

Prudente (Brazil). United States: Dr. P. Steiner (U.S.A.).

General Secretary:

Dr. II. F. Born (U.S.A.).

Assistant General Secretary: Dr. P. F. Denoix (France).

Treasurer:

Dr. P. R. Peacock (Great-Britain).

Chairmen of Commissions:

Cancer Research:

Prof. H. Druckrey (Germany).

Cancer Control:

Dr. Ch. S. Cameron (U.S.A.).

Chairmen of Committees:

ACTA:

Prof. J. H. Maisin (Belgium).

International Congresses:

Prof. R. Paierson (Great-Britain).

Elected Members:

Members serving one term (to be replaced Members serving two terms (to be replaced or re-elected at the Vllth Congress): or re-elected at the Vlllth Congress):

Dr. J.-P. Nicod (Switzerland). Prof. A. Prudente (Brazil). Dr. A. Rahausen (Chile). Dr. H. L. Stewart (U.S.A.). Dr. S. Tjokronegoro (Indonesia).

Dr. J. Clemmesen (Denmark). Dr. J. Gillman (South Africa). Prof. V. R. Khanolkar (India). Dr.

M. Puente-Duany (Cuba). Dr. 0. II. Warwick (Canada).

Members serving three terms (to be replaced

or re-elected at the IXth Congress):

Dr. N. Blokhine (U.S.S.R.).

Dr. C. Dukes (Great Britain).

Prof. //. Hamperl (Germany).

Prof. A. Lacassagne (Prance). 
Dr. G. T. Pack (U.S.A.).

Unio Internationalis Contra Cancrum

237

Commission de la recherche / Commission on Cancer Research

Chairman:

Pr. Hermann Druckrey, Hugstetterstrafie

55, Freiburg i. Br. (Germany).

Members:

Pr. /. Berenblum, Weizmann Institute of Science, Department of Experimental Biology,

Rehovoth (Israel). Dr. N. Blokhine, Academies des Sciences medicales de l'U.R.S.S., Salenka

14, Mos-cou (U.R.S.S.).

Dr. Harold F. Dorn, National Institutes of Health, Bethesda 14, Ma. (U.S.A.). Pr. Alexander

Haddoio, The Chester Beatty Aus Acta Unio Internationalis

Research Institute, Fulham Road, London S.W.3 (England).

Pr. Charles Huggins, The Ben May Laboratory for Cancer Research, The University of Chicago, 950, East 59th Street, Chicago 37, Illinois (U.S.A.).

Pr. V. R. Khanollcar, Tata Memorial Hospital, Parel-Bombay (India). Pr. A. Lacassagne, Institut du Radium, 26, rue d’Ulm, Paris (France). Dr. Cornelius Rhoads, Memorial Hospital, 444, East 68th Street, New York (U.S.A.). Pr. T. Yoshida, University of Tokyo, Medical School, Tokyo (Japan). Contra Cancrum XI (1955) 81.

International Committee on causative Factors of Cancer

Chairman:

Prof. Truhaut, Paris (France).

Members:

Prof. J. Berenblum (Israel)

Prof. F. Bielschowsky (New Zealand).

Prof. E. Boyland (Great Britain).

Dr. A. M. Brues (U.S.A.).

Prof. P. Ekwall (Finland).

Prof. Th. Gillman (South Africa).

Prof. A. Oraffi (Germany).

Prof. W. C. Hueper (U.S.A.).

Prof. M. Kuru (Japan).

Dr. R. Latarjet (France).

Dr. A. Nelson (U.S.A.).

Prof. P. R. Peacock (Scotland).

Prof. Serebrov (U.R.S.S.)

Prof. W. E. Smith (U.S.A.).

Dr. A. Tannenbaum (U.S.A.).

Prof. A. Timofeevski (U.R.S.S.).

Dr. A. L. Walpole (Great Britain).

i”. Sub-Committee on Ionising Radiation

Members:

Prof. L. Bugnard (France). Dr. J. Furth (U.S.A.). Prof. S. Hultberg (Sweden). Prof. H. S. Kaplan (U.S.A.). Prof. V. R. Khanolkar (India).

II. Sub-Committee on Air Chairman: Dr. Rhoads (U.S.A.). 
Members:

M. Cuzin (France). Prof. J. Firket (Belgium). Prof. P. Kotin (U.S.A.). Prof. Kreyberg (Norway). Prof. A. Lacassagne (France). Prof. II. Langendorfj (Germany). Dr. H. Lisco (U.S.A.). Dr. L. F. Lamerton (Great Britain)). Prof. B. Rajewsky (Germany). Prof. II. R. Schinz (Switzerland). Pollution and Tobacco Prof. Lickint (Germany). Dr. S. Neukomm (Switzerland). Prof. P.

Peacock (Great Britain). Dr. R. E. Waller (Great Britain). Prof. E. L. Wynder (U.S.A.). Prof. E. Kennaway (Great Britain). Dr. W. C. Hueper (U.S.A.).

238

Unio Internationalis Contra Cancrum

777. Sub-Committee on Food Additives

Chairman:

Prof. R. Truhaut (France).

Members:

Prof. E. Boyland (Great Britain).

Prof. H. Druclcrey (Germany).

Dr. A. R. Oopal-Ayengar (India).

Dr. 0. Hecht (Germany).

Dr. A. J. Lehman (U.S.A.).

Dr. A. Nelson (U.S.A.). Prof. A. Serebrov (U.R.S.S.). Prof. P. Shubik (U.S.A.). Prof. H.

Simmonet (Fance). Prof. D. 0. Steyn (South Africa). Dr. A. Tannenbaum (U.S.A.). Dr. M.

Umeda (Japan). Dr. H. Van Oenderen (Holland). Dr. R. T. Williams (Great Britain).

Committee on Clinical Stage Classification and Applied Statistics

C.C.S.C.A.S.

Chairman:

Dr. P. F. Denoiz (France).

Secretary:

M. 0. Roxy-Nobre (Brazil).

Members:

F. Baclesse (Fance).

R. Barajas-Vallejo (Mexico)

M. M. Copeland (U.S.A.). H. Hamperl (Germany). Sven Hultberg (Sweden). E. Lima-Basto (Portugal). W. P. D. Logan (Great Britain). R. McWhirter (Scotland). I. H. Perry (U.S.A.). A.I.Racov (U.R.S.S.). A. H. Sellers (Canada). 